See discussions, stats, and author profiles for this publication at: https://www.researchgate.net/publication/271484205

\title{
A novel approach to perform reversible addition/subtraction operations using deoxyribonucleic acid
}

Conference Paper in Proceedings - IEEE International Symposium on Circuits and Systems · June 2014

DOI: 10.1109/ISCAS.2014.6865513

CITATION

1

3 authors:

Ankur Sarker

University of Virginia

26 PUBLICATIONS 145 CITATIONS

SEE PROFILE

Md. Saiful Islam

Griffith University

74 PUBLICATIONS 588 CITATIONS

SEE PROFILE

Some of the authors of this publication are also working on these related projects:

Answering WHY and WHY-NOT Queries in Databases View project

Detecting Event Causality from Social Media Short Text View project

\section{READS}

64

Hafiz Md. Hasan Babu

University of Dhaka

88 PUBLICATIONS 817 CITATIONS

SEE PROFILE 


\title{
A Novel Approach to Perform Reversible Addition/Subtraction Operations Using Deoxyribonucleic Acid
}

\author{
Ankur Sarker ${ }^{1}$, Hafiz Md. Hasan Babu ${ }^{l}$ and Md. Saiful Islam ${ }^{2}$ \\ ${ }^{I}$ Dept. of Computer Science and Engineering, University of Dhaka, Dhaka 1000, Bangladesh \\ ${ }^{2}$ Dept. of Computer Science and Software Engineering, Swinburne University of Technology, VIC 3122, Australia \\ E-mail:ankur.sum@gmail.com,hafizbabu@cse.univdhaka.edu,mdsaifulislam@swin.edu.au.
}

\begin{abstract}
Reversible logic transforms logic signal in a way that allows the original input signals to be recovered from the produced outputs, has attracted great attention because of its application in many areas. Traditional silicon computers consume much more power compared to computing systems based on Deoxyribonucleic Acid (DNA). In addition, DNA-based logic gates are stable and reusable. In this paper, we propose a new approach for designing DNA-based reversible adder/subtractor circuit; it's possible to perform addition and subtraction operations using single circuit representation. We first merge the properties of addition and subtraction operations. Then, we demonstrate reversible DNAbased addition and subtraction operations. Our proposed DNAbased reversible addition/subtraction circuit is faster than the conventional one due to parallelism and replication properties of DNA strands. It also requires less space because of compactness of DNA strands. In addition, the DNA-based adder/subtractor circuit needs low power as the formation of DNAs consumes a small amount of energy. Finally, the comparative results show that the proposed DNA-based system requires $m+3.2^{n}$ DNA signals, but in existing system, it requires $m .2^{n}$, where $m$ is the size of extra tags and $n$ is the total number of bits. Besides, the run time complexity of proposed system has $O(1)$ while the existing system has $\mathrm{O}\left(\mathrm{mln}_{2} n\right)$.

Keywords- Reversible Logic, DNA and DNA Bases, DNA Annealing, Reversible Logic Gates, DNA Computing, Addition, Subtraction.
\end{abstract}

\section{Introduction}

In 1994, Adleman has solved the Hamilton Path Problem with 7-vertices using DNA [1]. The information inside the DNA are stored as a code made by four chemical bases: Adenine (A), Guanine ( $G)$, Cytosine (C) and Thymine (T). The bonding between two bases of DNA happens between complementary bases, $A$ with $T$ and $C$ with $G$, form base pair units [2]. There are also some non-natural bases like Xanthine $(X)$, Pseudouridine $(\Psi)$ and N(2)Dimethylguanosine (D) are complimentary to Adenine $(A)$. Scientists all over the world are now trying to exploit these unique features of DNA to make future computational system to overcome the present limitations of traditional silicon computers, also looking for the additional uneven features of DNA as well. It has been found that WatsonCrick hydrogen bonding is not the only way of interaction between the nucleobases in DNA. Rather, some other important $\mathrm{H}$-bonding interactions between nucleobases are Hoogsteen and Haschemeyer-Sobell interactions, aid to generate bonding between the natural bases and some nonnatural bases [3]. Among the non-natural modified bases, some are Inosine (I), Xanthosine (X), Pseudouridine ( $\Psi), 1$ Methylguanosine ( $\left.m^{l} G\right), \quad N(2)$-Dimethylguanosine (D). These modified bases are found to modulate some inherent functions of DNA like structural stability, temperature tolerance etc.

Reversible logic was introduced with a view to minimize the energy loss of a circuit. According to Landauer, in irreversible circuits, every bit of information loss generates $K T \ln 2$ Joules of energy, where $K$ is the Boltzmann constant of $1.38 \times 10^{-23} \mathrm{~J} / \mathrm{K}$ and $T$ is the operating temperature [4]. According to Bennet, zero energy dissipation would be possible only if the network consists of reversible gates [5]. As, reversible logic provides one-to-one mapping between the input and output vectors and ensures that the number of input vector is equal to the number of output vector. So, the mapping between input vector and output vector reduces the information loss.

Followings are the main advantages of using DNA-based computations over the existing silicon-based computations:

o Data density of double strands DNA will be one base per square nanometer and it will be over one million Gbits/square inch, where data density of typical high performance hard drive is about 7 Gbits per square inch [6-9].

o Base pair complementation gives a unique error correction mechanism as like RAID 1 array [10].

o As many copies of the enzyme can work on many DNA molecules simultaneously. It can work in a massively parallel fashion [11].

o DNA is a stable molecule that never suffers from any changes (mutation) unless it faces harsh (very high temperature, corrosive agents etc.) environment [19].

o Logic gates based on DNA can be preserved for a very long time (more than a decade) by maintaining and varying temperature [12], [13].

So, from the above discussion we can understand the DNA-based reversible operations require less space and it has self error-recovery capability. Moreover, it also gets parallelism and faster read-write capability.

The organization of this paper is as follows: Section 2 represents the prior knowledge associated with our proposed methods; Section 3 introduces the relation between 0/1 logic and DNA-based logic. Section 4 presents the concept of merging addition and subtraction operations, also, describes our methodology; besides, Section 5 depicts the comparative analysis. Finally, we conclude this paper with overall significances of our work with future possibilities.

\section{Basic Definitions and Properties}

In this section, we present the basic definitions and ideas related to reversible logic and DNA computing.

A $\boldsymbol{k} \times \boldsymbol{k}$ reversible gate is a $k$-input and $k$-output circuit that produces a unique output pattern for each possible input 
combination [15]. Let the input and output vector be $I_{V}$ and $O_{V}$, where $I_{V}=\left(I_{1}, I_{2}, \ldots, I_{k}\right), O_{V}=\left(O_{1}, O_{2}, \ldots, O_{k}\right), I_{V} \leftrightarrow O_{V}$ Fig 2.1 shows a $k \times k$ reversible gate. One bit inverter is a $1 \times 1$ reversible gate. Unused outputs of a reversible gate are known as garbage outputs, are only used to maintain the reversibility. When a Feynman Gate (FG) is used for Ex-OR operation of two inputs, extra output $P$ is added, this additional output is known as garbage output [15]. A $3 \times 3$ Toffoli gate realizes $P=A, Q=B$, and $\mathrm{R}=A B \oplus C$, where $A, B, C$ are inputs and $P, Q, R$ are outputs [14]. Similarly, A $3 \times 3$ Fredkin gate realizes $P=A, Q=A^{\prime} B \oplus A C$ and $R=$ $A^{\prime} C \oplus A B$, where $A, B$ and $C$ are inputs and $P, Q$ and $R$ are outputs [15].

$\boldsymbol{D N A}$ is the deoxyribonucleic acid. Two chains of DNA are in a right handed double helix. The strands are joint by hydrogen bonding between the bases of opposite strands, to form the base pairs [2]. DNA Denaturation, also known as DNA melting, is the process by which $d s D N A$ (doublestranded) unwinds and separates into single strands through the breaking of $H$-bonds between the bases and becomes sSDNA (single-stranded). Both terms are used to refer to the process as it occurs when a mixture is heated, although "DNA Denaturation" can also be referred to the separation of DNA strands induced by chemicals like urea. In DNA replication, DNA denaturation occurs among DNA and it transforms into single-stranded DNA. On the other hand, DNA Renaturation or annealing means the formation of dsDNA (double-stranded) from ssDNA (single stranded) with the help of base pairing by making H-bonds to make a complementary sequence. This term is often used to describe the reformation of complementary strands that were previously separated by heat (thermally denatured) or by use of some chemicals (chemically denatured). In final step of DNA replication, "DNA Renaturation" or annealing, also known as hybridization occurs by complementary bases formed using H-bonds. The 5'-end designates the end of the DNA or RNA strand that has the fifth carbon of the deoxyribose or ribose at its terminus where a phosphate group is attached. The 3'-end designates another end of the DNA or RNA strand and is so named due to its terminating at the hydroxyl group of the third carbon in the sugar ring.

III. Relation Between 0/1 Reversible Logic and DNA Several existing DNA-based designs have proposed the relationships between 0/1 logic with DNA logic [10], [16], [17]. However, existing DNA-based design [17] shows cleaver approach to encode 0/1 logic using two types of single DNA strands, one for input bit and another for operand bit. Also, in design [16], reversible Toffoli gate is realized using different DNA strands for control inputs and target inputs of Toffoli gate where control input signals are constructed using the dinucleotides $5^{\prime}-A G$ and $5^{\prime}-C T$ representing bit 1 and 0 , respectively. The target inputs are constructed with $5^{\prime}-\Psi A$ and $5^{\prime}-U A$ as bit 1 and 0 , respectively. Similarly, the final outputs are constructed using mixer of $3^{\prime}-A U, 3^{\prime}-A D, 3^{\prime}-A T$ and $3^{\prime}-A X$ representing bit $1,1,0$ and 0 , respectively.
IV. Proposed DNA-Based Adder/Subtractor Circuit A half-adder circuit performs addition operation between input variables $\mathrm{A}$ and $\mathrm{B}$, equations of the summation and carry are as follows: Sum $=A \oplus B$, Carry $=A B$. Similarly, fulladder circuit performs addition operation for input variables $\mathrm{A}, \mathrm{B}$ and $\mathrm{C}$, however, equations of summation and carry are as follows: $\operatorname{Sum}=A \oplus B \oplus C$, Carry $=A B \oplus A C \oplus B C=C$ $(A \oplus B) \oplus A B$. Unlike adder circuit, a half-subtractor circuit performs subtraction operation between input variables $A$ and B. Here, equations of borrow and difference are as follows: Diff $=A \oplus B$, Borrow $=\bar{A} B$. Similarly, full-subtractor circuit performs subtraction operation for input variables $A$, $\mathrm{B}$ and $\mathrm{C}$. Thus, equations of borrow and difference are as follows: Diff $=A \oplus B \oplus C$, Borrow $=\bar{A} B \oplus \bar{A} C \oplus B C=$ $C \overline{(A \oplus B)} \oplus \bar{A} B$. In fact, addition and subtraction operations are only differ for Carry and Borrow. Since Carry and Borrow signals are not being required at one time, we can easily merge these signals using one exclusiveor operation. The overall representation is depicted in Fig. 4.1. Four reversible DNA-based Toffoli gates with one DNA-based Fredkin gate are used to construct the proposed adder/subtractor circuit, (the university of Toffoli gate makes possible to realize any composite circuit). First, it calculates $A B$ and $\bar{A} B$; then, it performs $A \oplus B$. Finally, it generates Carry/Borrow. There are seven inputs with one control signal, $S_{0}$ for Fredkin gate and three outputs (Add/Sub, Carry/Borrow and $S_{0}$ ) with four garbages. To construct the DNA-based adder/subtractor circuit, we adopt the procedure, technical parameters of existing method [16]. As like that work, two single stranded DNA ( $s S D N A)$ molecules are used here as control inputs and another is used as target input. The final output is produced using two control input signals and generated with pre-designed ssDNA segments which binds with the target input and make it double stranded $(d s D N A)$. Here, we propose the slightly modified pre-designed DNA segments with a Poly$A$ tail, it helps this segment to get more molecular weight compared to the target input and this modification is necessary for the effective execution of operations. The two control inputs with the target input are represented by a string of bits where each bit is represented by a dinucleotide (two consecutive nitrogenous base of DNA). As like the previous work [16], control inputs, target input and final output are encoded in the same way (not shown here). The non-natural bases are used to make the design more efficient in terms of performance. Also, it is used to modulate the final output signals. After performing the operations, there are unchanged two input signals (two $s S D N A$ ) and one output signal (one $d s D N A$, consists of control input signal with a larger predesigned oligo). This $d s D N A$ is denatured for separation of the smaller strand by capillary electrophoresis. This smaller strand is again ready to be annealed with the modified input strand (having poly A tail and inosines, I, binds with all the bases and indicates no value after binding with the bases and used here for blocking one bit). Meanwhile, all other things are transferred to storage for reusing. This double strand is now ligated with another input (two base pairs long double 
strand DNA fragment that adds either 1 or 0 bit signal) on the opposite site of flanking Poly- $A$ nucleotide tail. This ligated double strand is the output for the first round of addition/subtraction operations. To make sequential circuit, it is necessary to form all input in single stranded form. To fan-out third output of proposed gate, a unique tag is used in target input. As a result, third output is formed in partially double strand where left part with unique tag is double stranded (representing output) and right part is single stranded (equivalent to output forming in control input) which will be used to fan-out.

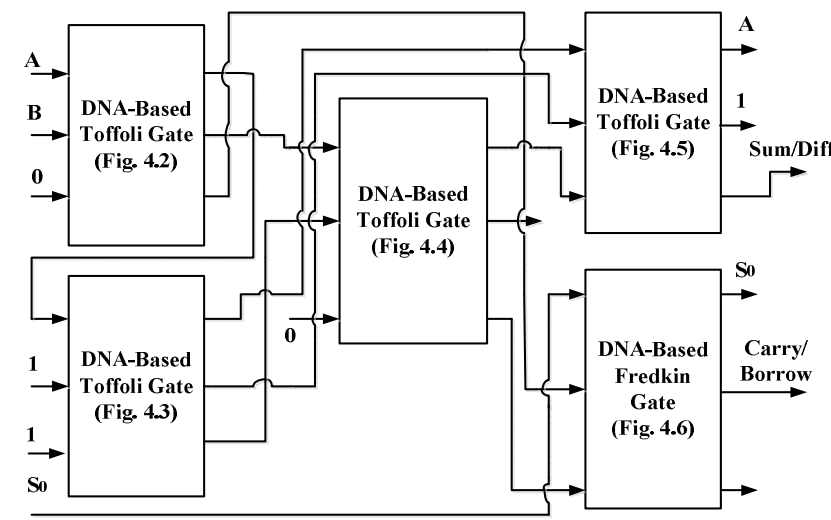

Fig. 4.1: Block diagram of the Hybridization process of the proposed DNA-based system for Addition/Subtraction Operation.

AGCTAGCT + CTAGAGCT + UAUAUAUA

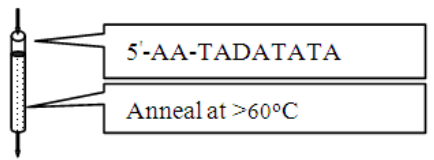

AGCTAGCT + CTAGAGCT +

UAUAUAUA

ATATADAT-AA

Fig. 4.2: DNA Hybridization of logical $A N D$ operation using DNA-based Toffoli gate.

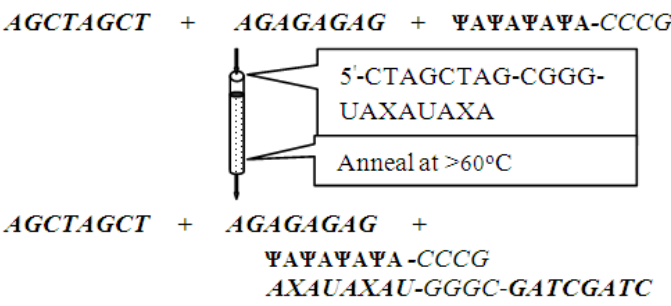

Fig. 4.3: DNA Hybridization of logical NOT operation using DNA-based Toffoli gate.

Later figures (Fig. 4.2-Fig. 4.6) show the sequential operaitons of proposed DNA-based half-adder/subtractor. Fig. 4.2 shows the operations of DNA-based Toffoli gate performing $A B$, where first input $\mathrm{A}$ represents 1010 and $\mathrm{B}$ represents 0110 ; the target output represents 0010 by using $3^{\prime}$ single strand DNA bases, then, it is fed into final Toffoli gate. Similarly, Fig. 4.3 shows the performing $\bar{A}$ operation, where final output is 0101 , also represented by $3^{\prime}$ single strand DNA bases, it is fed into middle Toffoli gate. Again, in Fig. 4.4, $\bar{A} B$ operation is performed using outputs of first and second Toffoli gates, produces 0100 represented by $3^{\prime}$
TCTCGATC which is fed into final Toffoli gate to produce Carry/Borrow signal.

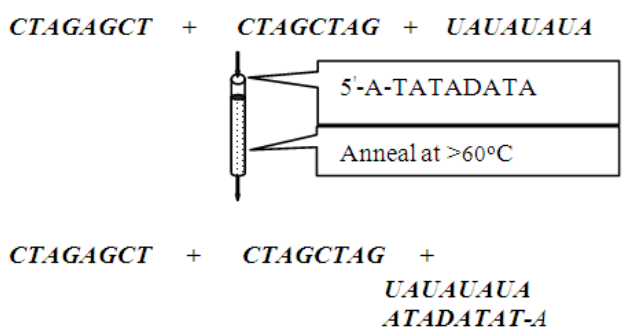

Fig. 4.4: DNA Hybridization of logical AND operation between complemented and non- complemented literals using DNA-based Toffoli gate.

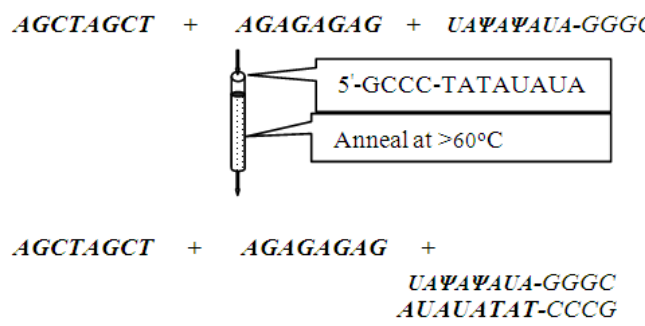

Fig. 4.5: DNA Hybridization of logical Ex-OR operation using DNA-based Toffoli gate.

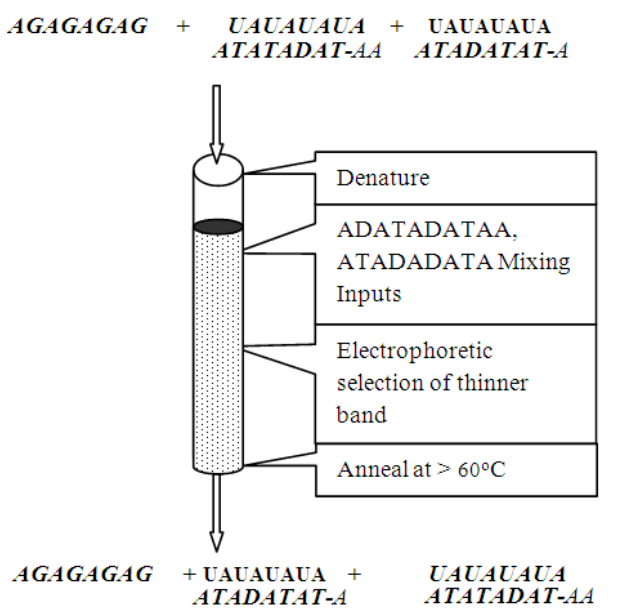

Fig. 4.6: DNA Hybridization of logical Ex-OR operation between two ANDed products using DNA-based Toffoli gate.

As like previous gates, Fig. 4.5 represents $A \oplus B$, the Add/Sub operation, here, the produced 3' single strand DNA represents 1100, which would be fed into fulladder/subtractor circuit. Finally, in Fig. 4.6, the selection operation of $A B$ and $\bar{A} B$, means the Carry/Borrow produced by control signal $S_{0}, 3^{\prime} A G A G A G A G$ single strand DNA bases which is fed into Fredkin gate. Now, to construct DNA-based reversible full-adder/subtractor circuit, we can easily use two half-adder/subtractor blocks with extra exclusive-or operations.

\section{Proposed Design Analysis}

In our proposed design, we use four DNA-based Toffoli gates and one Fredkin gate to construct overall circuit. For three inputs, total number of synthesizing DNA bases is $4 n$. In prior one [17], it had constraint of $m+2 n \leq 20$, but this 
system has no such constraint. However, we need to construct $m+3.2^{n}$ DNA signals, ( $\boldsymbol{n}$ is the total number of bits, $\boldsymbol{m}$ is the size of extra tags and 3 for considering inputs of Toffoli gate.) instead of $m .2^{n}$ [17]. We introduce 5 logic operations instead of 4 in prior design [17]. Also, a unit tag has to be generated for each operation which is not necessary in our proposed design. Besides, the probability of making new signal is $\frac{1}{2^{\mathrm{n}}}$. Also, in [17], denaturing and renaturing of DNA was necessary to make the output signal. Comparing with another existing DNA-based addition and subtraction operations [18], our proposed design performs better as that design used complex biological steps, 5 steps for addition and 6 steps for subtraction. For addition and subtraction operatoins, complexity of our design is $O(1)$ instead of $O\left(m \ln _{2} n\right)$ [18]. We summarize these discussions into Table I to compare our proposed design with others.

Table I: Comparison Between Existing DNA-Based Systems and the Proposed System.

\begin{tabular}{|c|c|c|}
\hline Parameters & $\begin{array}{c}\text { Existing DNA-Based } \\
\text { System [17] }\end{array}$ & Proposed System \\
\hline Constraint & $m+2 n \leq 20$ & None \\
\hline Required DNA signals & $m \cdot 2^{n}$ & $m+3.2^{n}$ \\
\hline $\begin{array}{c}\text { Total logical } \\
\text { operations }\end{array}$ & 4 logical operations & 5 logical operations \\
\hline $\begin{array}{c}\text { Maximum number of } \\
\text { making DNA signal }\end{array}$ & $2^{n}$ signal & Probability is $\frac{1}{2^{n}}$ \\
\hline $\begin{array}{c}\text { Process of DNA } \\
\text { formation }\end{array}$ & $\begin{array}{c}\text { Both denaturing and } \\
\text { renaturing are required }\end{array}$ & $\begin{array}{c}\text { Signals are used in } \\
\text { renatured form }\end{array}$ \\
\hline $\begin{array}{c}\text { Probability of } \\
\text { hydrolysis }\end{array}$ & Probability is high & Probability is low \\
\hline Signal types & Uniform & Not uniform \\
\hline $\begin{array}{c}\text { Complexity of DNA } \\
\text { formation }\end{array}$ & $\begin{array}{c}\text { Less DNA bases are } \\
\text { used, simple }\end{array}$ & $\begin{array}{c}\text { Few more DNA bases } \\
\text { are used, complex }\end{array}$ \\
\hline \hline Parameters & $\begin{array}{c}\text { Existing DNA-Based } \\
\text { System [18] }\end{array}$ & $\begin{array}{c}\text { Proposed System } \\
\text { Consists of complex } \\
\text { renaturing and } \\
\text { denaturing of DNA }\end{array}$ \\
\hline $\begin{array}{c}\text { Biological operations } \\
\text { bio-logical operations }\end{array}$ & $O\left(m \ln _{2} n\right)$ & $O(1)$ \\
\hline Run time Complexity & Ond \\
\hline
\end{tabular}

Here, $\boldsymbol{n}$ is the total number of bits and $\boldsymbol{m}$ is the size of extra tags.

From Table I, although there are few flaws (including extra tags, generalization) in our system, we can easily conclude our proposed system will perform better than other existing DNA-based designs [17], [18].

\section{Conclusion}

In this work, we realize the reversible adder/subtractor circuit using DNA-based inputs and outputs and the computations are performed using DNA. This study also aims to exploit some of these bases $(X, \Psi$ and $D)$ as well as natural bases to design a DNA-based composite circuit, they provide a broaden window of complementary to design the circuit. Also, some of these bases are used to modulate the final output signals by representing thyself mostly prioritized. So, whenever they are represented in output, the final signal will be the signal that they carried. This is one of the key characteristics of proposed DNA-based circuit. We also compare our proposed methodology with other DNA-based existing circuits. Since, to fan-out, extra bases are required, the demonstrated circuit outperforms the existing reversible logic gate in terms of speed, error correction, and power consumption. Unlike reversible logic gate, there is no use of silicon chip, only use of DNA bases. As a result, it is very faster [11] and lasts longer by maintaining and varying temperature [19]. Because of unchanging the input sequences, we get the reusability of it. Moreover, we also show that the proposed circuit has no constraint, requires fewer DNA signals and less complex in performing addition and subtraction operations than the existing DNA-based designs [17], [18].

\section{References}

[1] L. M. Adleman, "Molecular computation of solutions to combinatorial problems," Science-AAAS-Weekly Paper Edition, vol. 266, no. 5187, pp. 1021-1023, 1994.

[2] J. D. Watson and F. H. C. Crick, "Molecular structure of nucleic acids: a structure for deoxyribose nucleic acid," Annals of the New York Academy of Sciences-Paper Edition, vol. 758, no. 12, 1995.

[3] B. Liu, M. L. Wong and B. Alberts, "A transcribing RNA polymerase molecule survives DNA replication without aborting its growing RNA chain," Proceedings of the National Academy of Sciences, vol. 91, no. 22, pp. 10660-10664, 1994.

[4] R. W. Keyes and R. Landauer, "Minimal energy dissipation in logic," IBM Journal of Research and Development, vol. 14, no. 2, pp. 152-157, 1970.

[5] C. H. Bennettk, "Logical reversibility of computation," IBM journal of Research and Development, vol. 17, no. 6 pp. 525-532, 1973.

[6] D. M. H. Fulekar, "Bioinformatics: Applications in life and environmental sciences," Springer, pp. 200-206, 2009.

[7] W. Ryu, "DNA computing: A primer," ArsTechnica, 2000.

[8] Y. Yingwei, "DNA COMPUTING: DNA Computers VS. Conventional Electronic Computers", 2002.

[9] H. Echols and M. F. Goodman, "Fidelity mechanisms in DNA replication," Annual review of biochemistry, vol. 60, no. 1, pp. 477$511,1991$.

[10] L. Karl, "DNA computing: arrival of biological mathematics," The mathematical intelligencer, vol. 19, no. 2, pp. 9-22, 1997.

[11] H. Hiasa, and K. J. Marians, "Primase couples leading-and laggingstrand DNA synthesis from oriC," Journal of Biological Chemistry, vol. 269, no. 8, pp. 6058-6063, 1994.

[12] H. Hiasa, and K. J. Marians, "Initiation of bidirectional replication at the chromosomal origin is directed by the interaction between helicase and primase," Journal of Biological Chemistry, vol. 274, no. 38, pp. 27244-27248, 1999.

[13] S. Lund and J. Dissing, "Surprising stability of DNA in stains at extreme humidity and temperature," International Congress Series, vol. 1261, pp. 616-618, Elsevier, 2004.

[14] T. Toffoli, "Reversible computing," Springer Berlin Heidelberg, pp. $632-644,1980$

[15] E. Fredkin, and T.Toffoli, “Conservative logic," Springer London, pp. 47-81, 2002

[16] A. Sarker, T. Ahmed, S. M. Rashid, S. Anwar, L. Jaman, N. Tara, and H. M. H. Babu, "Realization of Reversible Logic in DNA Computing", in BIBE, 2011, pp. 261-265.

[17] V. Gupta, S. Parthasarathy, and M. J. Zaki, "Arithmetic and logic operations with DNA," in 3rd DIMACS Workshop on DNA Based Computers, 1997, pp. 212-220.

[18] F. de Santis and G. Iaccarino, "A DNA arithmetic logic unit," WSEAS Transactions on Biology and Biomedicine, vol. 1, no. 4, pp. 436-440, 2004.

[19] H. Clausen-Schaumann, M. Rief, C. Tolksdorf and H. E. Gaub, "Mechanical stability of single DNA molecules," Biophysical Journal, vol. 78, no. 4, 2000: 1997-2007. 